\title{
Genitourinary Applications of MR-Guided High-Intensity Focused Ultrasound
}

\author{
Fergus V. Coakley $\cdot$ Steven S. Raman • \\ Antonio C. Westphalen
}

Published online: 14 October 2014

(C) Springer Science+Business Media New York 2014

\begin{abstract}
Magnetic resonance-guided high-intensity focused ultrasound therapy is a novel and non-invasive treatment option for both benign and malignant tumors. In this article, we provide an overview of its basic technical features and biological effects, and describe the main genitourinary applications.
\end{abstract}

Keywords Genitourinary applications · High-intensity focused ultrasound $\cdot$ Magnetic resonance

\section{What is High-Intensity Focused Ultrasound?}

High-intensity focused ultrasound (HIFU) refers to a noninvasive thermal ablation technique that uses tightly focused high-energy ultrasound waves to generate heat and ultimately destroy tissue by coagulative necrosis. Each thermal dose delivery, called sonication, treats a sharply delineated fraction of the targeted tissue. And overlapping multiple sonications, one treats an entire volume. The procedure is usually performed under sedation and/or regional anesthesia, i.e., nerve block or spinal anesthesia. Yet, HIFU is well tolerated, and most patients are discharged home after a few hours of observation with only mild pain management.

One of the initial publications describing its use in animal experiments dates back from 1954 [1], but the technique has since evolved significantly. It is currently approved in many countries for the treatment of uterine leiomyomas. In

This article is part of the Topical Collection on Urogenital Imaging.

All authors contributed equally to the preparation of this manuscript.

F. V. Coakley · S. S. Raman · A. C. Westphalen ( $₫)$

University of California, San Francisco, USA

e-mail: AntonioCarlos.Westphalen@ucsf.edu addition, many research projects are underway to determine other genitourinary applications, including the treatment of uterine adenomyosis and prostate cancer.

In the vast majority of the cases, HIFU is used in conjunction with an imaging modality, ultrasound, or magnetic resonance (MR) imaging, to identify the target and direct the high-energy ultrasound beam.

\section{Why MR-Guided High-Intensity Focused Ultrasound?}

\section{Improved Thermometry}

Ultrasound cannot estimate the thermal dose that is given during treatment. Instead, the assessments of its effects are based on changes in tissue echogenicity, which are mostly secondary to microbubble formation, and reflect an energy in excess of that needed for an adequate ablation $\left(100{ }^{\circ} \mathrm{C}\right)$. In contrast, MR guidance allows for an accurate near realtime temperature reading using a multi-slice MR imaging $3 \mathrm{D}$ temperature map of the treated region. The MR-guided HIFU thermometry system calculates the thermal dose and indicates whether it is sufficient for coagulative necrosis. This allows to confirm that heating is limited to the planned region, and to monitor any unintentional, non-damaging heating elsewhere in the ultrasound beam path, letting the radiologist change of the treatment plan accordingly. While a thermal dose of $60{ }^{\circ} \mathrm{C}$ is sufficient to cause cellular death [2], in most cases, doses of $65-85{ }^{\circ} \mathrm{C}$ are delivered for a few seconds to guarantee adequate treatment.

Improved Sonication Planning

The device used for MR-guided HIFU ablations has multiple individual elements in the transducer, allowing for 
electronical steering of the beam during treatment. More importantly, however, is the ability to generate treatment volumes of various shapes and sizes that precisely conform to the anatomical target. This is analogous to intensitymodulated radiation therapy, in which the radiation beam conforms to the three-dimensional shape of the treatment site, allowing for the delivery of higher doses without increasing the dose to adjacent tissues.

\section{MR-Guided High-Intensity Focused Ultrasound for Uterine Leiomyomas}

Uterine leiomyomas, also called fibroids or myomas, are benign smooth muscle estrogen sensitive tumors of variable size present in up to $70 \%$ of women by age $45[3,4]$. They are generally asymptomatic but may cause either bulk-related symptoms such as pelvic pain, pressure or urinary frequency, or endometrial pressure symptoms such as heavy vaginal bleeding (menorrhagia) leading to anemia [5]. The overall type and severity of leiomyoma-related symptoms may be quantified by a validated, standardized questionnaire, the Uterine Fibroid Symptom and Health-Related Quality of Life (UFS QOL) survey. This tool may be used to categorize and quantify patient's bulk, bleeding, and related symptoms [6]. Medical options for relief of these symptoms are temporary. Definitive treatment usually involves open surgical myomectomy and hysterectomy, with increasing use of less invasive laparoscopic or robotic hysterectomy or myomectomy in selected centers. A minimally invasive alternative, uterine artery embolization, selectively and temporarily cuts off blood supply to one or more uterine leiomyomas resulting in infarction-related decreases in leiomyoma volume and a change in composition. However, it is currently not recommended for women who desire future fertility and does have a small risk of inducing ovarian failure and premature menopause in some women. MR-guided HIFU is a novel non-invasive technology approved for treatment of uterine fibroids. Two systems are currently available worldwide: The ExAblate system from Insightec (Insightec Inc. Haifa, Israel) is compatible with GE MR systems (GE Healthcare Milwaukee, Wi) and the Sonalleve system (Philips, Vantaa, Finland) from Philips is compatible with Philips MR systems (Philips Healthcare, Best, Netharlands). A wide variety of ultrasound (US)-guided HIFU systems are available mainly in Asia and Europe, but this article will focus on the MR systems.

Diagnosis of Uterine Leiomyomas: The Role of Imaging

US is the initial modality of choice in the diagnosis of uterine fibroids and is often performed in the primary care physician's office to establish a diagnosis. On US, uterine fibroids are discrete round or oval lesions of variable echogenicity (typically hypoechoic) often with calcifications located in intramural, subserosal, or submucosal locations. Intracavitary lesions projecting into the endometrial canal and pedunculated lesions projecting into the peritoneal cavity may be seen. However, US has been shown to be too operator dependant to determine the size and number of lesions especially in patients with multiple lesions and cannot determine the composition of lesions or the presence of adenomyosis. MR imaging overcomes many of these limitations [7]. It is reproducible with excellent soft tissue contrast and multiplanar capability and enables assessment of uterine anatomy, fibroid size, and composition. Uterine fibroids are either classical or hypercellular and may undergo degeneration. Both classical and hypercellular myomas are typically isointense to the uterine myometrium on T1-weighted scans. On T2weighted scans, classical myomas are hypointense to myometrium, and hypercellular myomas are hyperintense to myometrium. Both types of lesions enhance avidly on contrast-enhanced T1-weighted images. Uterine myomas may spontaneously undergo either cystic or red degeneration. Cystic degenerated myomas are variably T2 hyperintense, while red degenerated myomas are typically T2 hypointense and mildly T1 hyperintense. Both types of lesion do not enhance after intravenous contrast administration.

\section{Management of Patients with Uterine Leiomyomas}

\section{Medical Therapy}

Medical therapy consists of a variety of short-term options for controlling both bulk and bleeding symptoms. These include oral contraceptives for control of uterine bleeding and non-steroidal anti-inflammatory drugs (NSAIDS) for menstrual pain (dysmenorrhea). GnRH agonists such as leuprolide acetate (Lupron) lead to a 35-65 \% reduction of fibroid volume and are approved for the preoperative treatment of women with anemia with temporary improvement of bulk symptoms [8]. Treatment is typically recommended for up to 6 months. Longer term treatment would lead to an unacceptably high side-effect profile due to hypoestrogenic state.

\section{Surgical Therapy}

A variety of surgical therapies remain the most definitive treatment for uterine fibroids. Hysterectomy is the main option for family complete women and may be performed open, laparoscopically, robotically, or vaginally. Up to 200,000 hysterectomies are performed annually in the 
United States at a cost of over \$2 billion. However, recovery may last for 1-5 weeks, and certain long-term complications such as pelvic prolapse are reportedly higher in women undergoing hysterectomy [9, 10]. A significant subset of women prefers less invasive procedures and for these women, evaluation in a multidisciplinary practice may be preferred [11]. For women who choose to preserve their uterus, myomectomy performed by open, laparoscopic, robotic-assisted, or hysteroscopic methods is a surgical option that has been proven to be safe and effective for relief of both bleeding and bulk symptoms [12-16]. However, myomectomy does have a variably high risk of fibroid recurrence, and complications such as uterine rupture have been reported.

\section{Uterine Artery Embolization}

Uterine artery embolization (UAE) was initially described for treatment of uterine leiomyomas in Europe in 1995 [17] and in the US in 1999 [18]. In this procedure, embolic particles such as poly vinyl alcohol of trisacryl gelatin microspheres or beads are injected into the uterus via femoral artery catheterization and selective placement into both uterine arteries. Embolization cuts off of blood flow to dominant vessels of individual fibroids resulting in selective ischemic necrosis of individual fibroids with sparing of uterine myometrium due to its rich collateral blood supply. These necrotic embolized leiomyomas progressively involute with a volume reduction of up to $40 \%[19,20]$. UAE has consistently demonstrated excellent short- and long-term control of both bleeding and bulk symptoms in multiple studies, as shown in a recent large meta-analysis [21]. Further, there have UAE has been shown to be safe and effective compared to both hysterectomy [22] and myomectomy [23] in randomized controlled trials with significantly shorter hospital stays, faster recovery, and similar morbidity.

\section{MR-Guided High-Intensity Focused Ultrasound}

MR-guided focused US also known as MR-guided focused US surgery (MRgFUS) is an innovative, non-invasive application of HIFU to treat small to intermediate uterine fibroids [24-26, 27••]. The ExAblate system (Insightec Inc., Haifa, Israel) is the only commercially approved HIFU system by the U.S. Food and Drug Administration (F.D.A.). The Philips Sonalleve system and three different US-guided HIFU systems are approved outside the United States in Asia and Europe [28]. All patients considering MRgFUS must have no contraindications to MR Imaging and be able to lie prone on the HIFU table for up to $3 \mathrm{~h}$. Generally, indications for MRgFUS include non-pedunculated, non-degenerated, non-peripherally calcified, T2 hypointense fibroids generally below $500 \mathrm{cc}$. Larger lesions may be shrunk with GnRH analogs for up to 6 months or less optimally be treated in multiple sessions since there is an F.D.A. mandated individual treatment time limit of $3 \mathrm{~h}$. Although initially contraindicated for women who were considering childbearing, the F.D.A. changed this to a relative contraindication in 2009.

To be eligible for MRgFUS, patients must not have any contraindications to 1.5 or 3T MRI. Metallic implants or scars traversing the lower anterior pelvic wall in the path HIFU beam may lead to unintended heating and skin burn. Prior to MRgFUS, all anterior pelvic wall hairs above the pubic bone are shaved to avoid trapping of air bubbles in the path of the US beam. After placement of a Foley catheter and an intravenous line, the patient lies prone on a dockable table on a degassed water bath containing a gel pad overlying the MR compatible HIFU transducer. The procedure is typically performed with intravenous conscious sedation with anxiolytics and narcotics. A T2weighted sequence is performed in three planes and registered to register with the HIFU unit. After ensuring that no bowel lies between the skin and target fibroid, a treatment volume is graphically plotted on to target fibroid for both systems [24-26, 27••, 28, 29].

In the Insightec ExAblate system, a treatment plan is generated with a series of ovoid, cigar-shaped zones of sonications of user-controlled length $(1-4 \mathrm{~cm})$ and width $(0.5-1 \mathrm{~cm})$. In the planning phase, test sonications are initially delivered to assure that target heating occurs with proper alignment. Intermittent proton resonance frequency imaging is used to generate tissue temperature mapping for MR thermometry to assure that sonicated area reaches a minimum of $60 \mathrm{C}$ necessary for coagulative necrosis. In the treatment phase, based on a semi-automated algorithm, the Exablate software selects individual sonications, and coagulative US energy is delivered for $20 \mathrm{~s}$ followed by a 90-120 s interval ("cooling duration" to minimize skin heating). Up to 120 such sonications are delivered in one session with each sonication is projected over an anatomic image. The cooling duration accounts for the majority of treatment time [24-26, 27••, 28, 29].

In the Philips Sonalleave system, a 3D T2 turbo spin echo is performed and used for treatment planning. The planned treatment volume (PTV) is graphically prescribed by the treating radiologist. Instead of point by point sonications, a series of enlarging clockwise, concentric sonications with progressively enlarging sonication diameters radiate out from the center of the PTV. Preliminary studies have shown the system to be safe with agreement between planned ablation volumes and post-procedural imaging and histopathology [30, 31].

The widespread acceptance of MRgFUS by gynecologists and payors has been limited by lack of randomized 
controlled trial data, relying instead on single-arm industry sponsored trials. Initial reports were primarily safety trials which demonstrated that the procedure could be performed safely on an outpatient basis with minimal morbidity. These studies also demonstrated a small but significant improvement in symptoms on validated symptom severity questionnaires even in incompletely treated leiomyomas $[24,25]$. Further studies demonstrated a very favorable safety profile for treatment of larger leiomyomas and also demonstrated that more effective and sustained response for bleeding and bulk to 2 years was possible when larger volumes $(>60 \%)$ of individual leiomyomas were treated $[26,27 \bullet \bullet]$. The current goal for fibroid treatment is to near completely treat the largest lesions for relief of bulk symptoms and to treat submucosal lesions for relief of menorrhagia. In general with current technology, a single 7-8 cm classical leiomyoma or 3-4 smaller classical leiomyomas with similar total volume may be treated in one 3-hour session. Larger leiomyoma volumes of lesions may require more than one session or require a three-month course of GnRH analog therapy to decrease leiomyoma volume.

More recent studies have confirmed initial studies and have established that complete or near complete ablation of uterine leiomyomas provides the best long short- and longterm symptom relief with up to $88 \%$ overall symptom improvement at 12 months [29]. Further with newer technologies, higher NPV ratios have become routinely feasible in clinical practice with minimal adverse events [32]. A $41 \%$ HIFU live pregnancy and $28 \%$ spontaneous abortion rate have been reported after HIFU [33••].

\section{MR-Guided High-Intensity Focused Ultrasound for Uterine Adenomyosis}

Adenomyosis is a benign entity characterized by the presence of ectopic endometrial glands and stroma within the myometrium, which is hypertrophic and hyperplastic, resulting in an enlarged and softened uterus [34]. It is a common disease, affecting approximately $20-30 \%$ of adult women [35]. More importantly, though, two-thirds of these women present with menometrorrhagia, dysmenorrhea, dyspareunia, pelvic pain, and possibly infertility [36].

\section{Diagnosis of Adenomyosis: The Role of Imaging}

Once clinically suspected, additional work-up relies heavily on imaging. On transvaginal ultrasound, the uterus is enlarged; the myometrium is typically heterogeneous, asymmetric, and globular, with linear striations and microcysts; the junctional zone is poorly visualized; and there is no mass effect on the endometrium. A recent meta- analysis showed that its sensitivity and specificity are 0.79 (95\% CI $=0.75-0.83)$ and $0.85(95 \% \mathrm{CI}=0.82-0.87)$, respectively [37]. MR imaging, however, is more specific than ultrasound $(0.91 ; 95 \% \mathrm{CI}=0.88-0,93)$ [37]. It is characterized by thickening of the junctional zone $>=12 \mathrm{~mm}$, and the presence of myometrial foci of high signal intensity on $\mathrm{T} 2$-weighted images.

Management of Patients with Adenomyosis

\section{Medical Therapy}

Treatment of patients with adenomyosis is mostly dependent of the severity of symptoms and desire to preserve fertility. In most cases, medical therapy represents the first line of treatment. Oral progesterones may be effective in treating pain and dysmenorrhea, but the levonorgestrelreleasing intrauterine device $\left(\right.$ Mirena ${ }^{\circledR}$ ) is considered a better option. Approximately, $20 \%$ of women on medical therapy will need further treatment, though [38]. And if pregnancy is desired, the treatment must be interrupted and symptoms are likely to return.

\section{Embolization and Surgical Therapy}

For patients who fail medical treatment, embolization of the uterine arteries and surgical procedures are common management alternatives. Uterine arteries embolization shows good results controlling menometrorrhagia in the short term, but approximately $25-50 \%$ of the patients will have symptoms relapse and require further treatment within 2 or 3 years $[39,40]$. Women who opt for surgery may undergo total hysterectomy or conservative resections, the latter usually reserved for cases in which preservation of fertility is required and adenomyosis is superficial and focal. Pregnancy rates after uterine-sparing procedures range between 50 and $60 \%$ [41]. Furthermore, complete symptomatic control is achieved in only about $30-50 \%$ of patients [41].

\section{High-Intensity Focused Ultrasound}

The use of HIFU to treat adenomyosis is relatively recent, and the published data are sparse. The technique has been shown to be safe and effective in controlling the symptoms of dysmenorrhea associated with focal and diffuse adenomyosis [42]. Additionally, it can be used to treat both superficial and deep adenomyosis, as opposed to other uterine preserving modalities. Similar to what happens with uterine leiomyomas, MR-guided HIFU takes advantage of the improved conspicuity of adenomyosis of MRI to achieve more accurate targeting and controlled delivery of thermal dose. In 2008, Fukunishi et al. published an initial 
series of women with symptomatic adenomyosis treated with MR-guided HIFU [43], and later in 2011, 2-year follow-up results were presented [44•]. In their cohort of 39 women, MR-guided HIFU achieved significant, sustained, and continuous control of symptoms. At about the same time, Fan et al. published their preliminary results in which women also conveyed significant improvement in quality of life and no significant complications after treatment [45]. While not definite, the data from these studies suggest that MR-guided HIFU should be considered as an alternative for the treatment of symptomatic adenomyosis.

\section{MR-Guided High-Intensity Focused Ultrasound for Prostate Cancer}

The concept of treating only the dominant disease site in the prostate, and not the entire gland, is the primary driving force behind the use of MR-guided HIFU for prostate cancer. Focal therapy for prostate cancer is an emerging paradigm that offers a "middle way" of management between active surveillance and definitive local therapy [46-48]. Focal therapy challenges the traditional view that prostate cancer is a multifocal disease that requires a whole-gland treatment approach for definitive local therapy, by either radical prostatectomy or radiotherapy. These treatments are associated with distressingly high side effects (including impotence, incontinence, and anorectal dysfunction) [49], particularly when weighed against the often modest and delayed survival benefits [50]. Such considerations have been partially responsible for increasing interest in focal therapy, but it is important to also review the biological rationale that underlies this emerging strategy, since several other factors, in addition to the desire to reduce treatment morbidity, have driven this development.

\section{Biological Rationale for Focal Therapy of Prostate Cancer}

\section{New Understanding of the Biological Significance of Prostate Cancer Multifocality}

Most studies report a multifocality rate for prostate cancer of around $80 \%$ [51]. The biological importance of multifocality is a central concern for focal therapy, since failure to treat an aggressive tumor focus might result in an adverse patient outcome. Fortunately, we now know that most non-index tumors are small, $80 \%$ are less than $0.5 \mathrm{~cm}^{3}$ [52], organ-confined, and of low Gleason score. Only two secondary foci were associated with extra-capsular extension and only one had a Gleason score greater than 6 in a series of 170 secondary foci found in 100 prostatectomy specimens [53]. In outcome studies, secondary foci have no incremental adverse prognostic significance over and above the index lesion [54-56]. Finally, there is reasonable evidence that multifocality may be a subjective observation. In two studies with similar populations undergoing radical prostatectomy at the University of California, San Francisco, the mean number of tumor nodules per patient varied from 3.4 (300 nodules in 88 patients) to 1.6 (58 nodules in 37 patients) $[57,58]$. The tumor size distribution suggests that the most plausible explanation is that the studies involved two different histopathologists. The primary difference was the percentage of very small nodules $\left(\leq 0.05 \mathrm{~cm}^{3}\right)$ detected by one pathologist versus the other $(60$ vs. $12 \%)$. That is, the frequency of multifocal prostate cancer may be largely dependent on the search intensity of the reviewing pathologist. This is analogous to breast cancer, where the rate of multifocality depends on how hard one looks; in clinical studies, the multifocality rate is typically about $9 \%$ [59], but rises to 54-63\% with more exhaustive histopathological techniques $[60,61]$.

\section{Oncological Insights into Index Lesion}

In parallel with the empirical observations on the limited importance of prostate cancer multifocality, advances in basic research have led to increasing emphasis on the biological importance of the index lesion, and the potential for focal therapy directed to the index lesion [62]. Direct empirical support of this concept is provided by recent data indicating that local recurrence of prostate cancer within the gland is nearly always at the site of initial dominant disease [63]. This supports the notion that more aggressive therapy directed to the dominant disease site may result in better local control.

\section{Development of MRI-Targeted Biopsy}

The ability to accurately localize dominant disease within the prostate with multiparametric endorectal MRI, particularly with the addition of diffusion-weighted imaging, has prompted exploration of MRI-targeted biopsy as an alternative to the traditional transrectal ultrasound-guided systematic biopsy, which essentially is a blind random sampling of the prostate. While the role of targeted biopsy, whether performed directly ("in-bore") or by a hybrid MRI/ultrasound technique ("fusion"), remains under investigation, it is conceivable that someday, at least in patients with a visible target, "MRI-guided biopsy will replace TRUS-guided biopsy" [64]. Targeted biopsy for disease confirmation and localization is clearly a critical pre-requisite for focal therapy; the technology exists to do this and simply requires greater validation before entering mainstream practice. 


\section{Patient Selection and Target Definition}

Selecting patients who are good candidates for focal therapy is challenging, given the spectrum of prostate cancer aggressiveness. Conceptually, at the low-risk end of the spectrum, patients with indolent or subclinical disease should be offered active surveillance, while those at the high-risk end should be managed with radical prostatectomy or definitive radiotherapy. Focal therapy, when provided in isolation, would likely work best for those with intermediate-risk disease. An intriguing question is whether focal therapy to the dominant disease site, when provided in combination with definitive radiotherapy, offers any incremental benefit in higher risk patients. Developing practical risk-based stratification schemes for the use of focal therapy is an important clinical question and represents a non-trivial challenge [65].

Second, assuming that appropriate candidates can be clinically selected and independent of the method of tissue ablation, any form of focal therapy for prostate cancer requires that the cancer is correctly identified and precisely ablated during therapy [66]. This is primarily a radiological problem. While not perfect, multiparametric endorectal MRI supplemented by biopsy is the best available technique to map and confirm the dominant tumor site. MR-guided HIFU seems best suited to patients with a visible dominant tumor at MRI, so that treatment is delivered to a tumor depicted by the same modality being used to guide therapy. This avoids the potential inaccuracies in "blindly" treating a designated portion of the prostate based purely on positive biopsies. Ideally, MR targets would be identified with high sensitivity and specificity. At a minimum, it seems reasonable to require that dominant tumor sites should be identified with high certainty, so that "false positive" tumors and the risk of not treating the true site of dominant disease are avoided. A recent study of patients undergoing endorectal multiparametric MRI prior to radical prostatectomy $(n=88)$ demonstrated that all T2-weighted tumor targets were correctly identified dominant tumor foci when associated with at least $0.54 \mathrm{~cm}^{3}$ of concordant spectroscopic imaging abnormality [58]. A subsequent study $(n=20)$ employing this definition of target tumor showed that all 9 tumor foci independently identified by both readers were correctly identified dominant tumor sites, but 5 lesions identified by only one reader were all false positives [67]. This suggests that identification of treatable tumor foci prior to MR-guided HIFU for prostate cancer should require both multiparametric and independent multi-reader confirmation.

Finally, focal therapy logically requires that a margin should be drawn around the target to ensure all of the intended tumor tissue that is actually included in the treatment volume. A study utilizing multiparametric MRI data and quantitative analysis of imaging contour compared to whole-mount histopathology adequate tumor coverage is achieved by expanding the treatment contour at the non-capsular margin by $5 \mathrm{~mm}$, with no expansion required at the capsular margin [67].

\section{Current status and Future Directions}

The first commercial HIFU systems for prostate ablation (Sonablate 100, Focus Surgery, Inc., Milipitas, CA, and Ablatherm, EDAP TMS Inc., France) utilized ultrasound for guidance. Early trials have suggested that ultrasoundguided HIFU can be successfully used to treat prostate cancer, with encouraging results related to local tumor control $[68,69 \bullet, 70,71]$; the results with respect to treatment morbidity are less promising. Rectourethral fistula has been reported in $0.7-1.2 \%$ of patients, impotence in $20-61 \%$, and incontinence in $0.6-14.6 \%$ [72]. These complication rates appear unacceptably high for a treatment that is intended to be minimally invasive and of low morbidity, and almost certainly reflect inadequate and imprecise targeting, resulting in unintended damage to the urethra, neurovascular bundles, or rectum. MRI provides superior visualization of the neurovascular bundles and the urethra, and provides real-time thermometry during a sonication, greatly reducing the risk to these important structures. Real-time MR thermometry and the inherent stereotactic nature of MR-guided HIFU are further major advantages that should improve technical performance. While there are potential benefits to MRI-guided HIFU, it is important to realize that there will also be risks. Based on experience with US-guided HIFU, potential complications include bladder neck/urethral stricture, urinary tract infection, urinary incontinence, urinary retention, impotence, rectal burn, or rectourethral fistula [73].

The two commercially available MR-guided HIFU systems are Sonalleve (Philips Healthcare, Vantaa, Finland) and ExAblate (InSightec, Haifa, Israel), and these two vendors are taking different approaches to prostate HIFU. The Insightec system uses an endorectal ultrasound transducer, while the Philips system uses a urethral device. Arguments can be made for and against both approaches. The rectal approaches may provide greater geometric flexibility and shaping of the target volume. The urethral approach may be faster and less likely to injure the rectum, but may not be as flexible with respect to working around the neurovascular bundles. It may be that both approaches should be available, with the final choice of a rectal or urethral approach being made depending on the location and shape of the tumor target in each individual patient. Early results suggest that MRI-guided HIFU of prostate cancer can be technically successful $[74,75 \bullet \bullet]$, but clearly 
studies with larger populations and longer cancer-based endpoints are required to validate this treatment method.

To date, most data on patient outcome after HIFU of prostate cancer are derived from populations treated with ultrasound-guided HIFU, with reported five-year biochemical control rates from 45 to $84 \%$ [73]. Longer term studies looking at hard endpoints (development of metastatic disease and mortality) will be required to determine the true effectiveness of this approach, either as monotherapy for localized low-risk disease or as combination therapy in conjunction with radiotherapy for higher risk disease. Animal data suggest that pre-treatment with HIFU may be a potent sensitizer for chemoradiation $[76,77]$.

\section{Conclusion}

MRI-guided HIFU is an exciting new method of precisely targeted and controlled tissue ablation that offers a minimally invasive therapeutic option for patients with uterine leiomyomas, uterine adenomyosis, and prostate cancer. The technology is potentially disruptive of current clinical practice patterns, and so wider adaptation will require multidisciplinary and multicenter investigations resulting in data demonstrating long-term efficacy and cost-effectiveness, in order to convince referring providers and third party payers that the approach should be added to our routine clinical armamentarium. Despite these challenges, the emergence of MRI-targeted focal therapy seems likely to be an important translational effort in the coming years, which promises to improve the personalized management of this challenging disease.

\section{Compliance with Ethics Guidelines}

Conflict of Interest Dr. Fergus V. Coakley, Dr. Steven S. Raman, and Dr. Antonio C. Westphalen each declare no potential conflicts of interest.

Human and Animal Rights and Informed Consent This article does not contain any studies with human or animal subjects performed by any of the authors.

\section{References}

Recently published papers of particular interest have been highlighted as:

- Of importance

•- Of major importance

1. Lindstrom PA. Prefrontal ultrasonic irradiation-a substitute for lobotomy. A.M.A. Arch Neurol Psychiatry. 1954;72(4):399-425.

2. Chu KF, Dupuy DE. Thermal ablation of tumours: biological mechanisms and advances in therapy. Nat Rev Cancer. 2014;14(3):199-208.
3. Okolo S. Incidence, aetiology and epidemiology of uterine fibroids. Best Pract Res Clin Obstet Gynaecol. 2008;22(4):571-88.

4. Marshall LM, et al. Variation in the incidence of uterine leiomyoma among premenopausal women by age and race. Obstet Gynecol. 1997;90(6):967-73.

5. Buttram VC Jr, Reiter RC. Uterine leiomyomata: etiology, symptomatology, and management. Fertil Steril. 1981;36(4): 433-45.

6. Harding $G$, et al. The responsiveness of the uterine fibroid symptom and health-related quality of life questionnaire (UFSQOL). Health and quality of life outcomes. 2008;6:99.

7. Levens ED, et al. Magnetic resonance imaging and transvaginal ultrasound for determining fibroid burden: implications for research and clinical care. Am J Obstet Gynecol. 2009;200(5): 537 e1-7.

8. Golan A, et al. D-Trp-6-luteinizing hormone-releasing hormone microcapsules in the treatment of uterine leiomyomas. Fertil Steril. 1989;52(3):406-11.

9. Flynn M, et al. Health care resource use for uterine fibroid tumors in the United States. Am J Obstet Gynecol. 2006;195(4):955-64.

10. Hartmann KE, et al. Annual costs associated with diagnosis of uterine leiomyomata. Obstet Gynecol. 2006;108(4):930-7.

11. Iverson RE Jr, et al. Relative morbidity of abdominal hysterectomy and myomectomy for management of uterine leiomyomas. Obstet Gynecol. 1996;88(3):415-9.

12. Vercellini $\mathrm{P}$, et al. Abdominal myomectomy for infertility: a comprehensive review. Hum Reprod. 1998;13(4):873-9.

13. Lee DW, et al. Uterine fibroid treatment patterns in a population of insured women. Fertil Steril. 2009;91(2):566-74.

14. Wang CJ, et al. Laparoscopic myomectomy for large uterine fibroids. A comparative study. Surg Endosc. 2006;20(9):1427-30.

15. Advincula AP, et al. Preliminary experience with robot-assisted laparoscopic myomectomy. J Am Assoc Gynecol Laparosc. 2004;11(4):511-8.

16. Jenkins SA, et al. Randomized double-blind placebo-controlled trial of early octreotide in patients with postoperative enterocutaneous fistula. Br J Surg. 1995;82(11):1576.

17. Ravina JH, et al. Arterial embolisation to treat uterine myomata. Lancet. 1995;346(8976):671-2.

18. Goodwin SC, et al. Uterine artery embolization for the treatment of uterine leiomyomata midterm results. J Vasc Interv Radiol. 1999;10(9):1159-65.

19. Spies JB, et al. Long-term outcome of uterine artery embolization of leiomyomata. Obstet Gynecol. 2005;106(5 Pt 1):933-9.

20. Pron G, et al. The ontario uterine fibroid embolization trial. Part 2. Uterine fibroid reduction and symptom relief after uterine artery embolization for fibroids. Fertil Steril. 2003;79(1):120-7.

21. Gupta JK, et al. Uterine artery embolization for symptomatic uterine fibroids. Cochrane Database Syst Rev. 2006;1:CD005073.

22. Hehenkamp WJ, et al. Uterine artery embolization versus hysterectomy in the treatment of symptomatic uterine fibroids (EMMY trial): peri- and postprocedural results from a randomized controlled trial. Am J Obstet Gynecol. 2005;193(5):1618-29.

23. Goodwin SC, et al. Uterine artery embolization versus myomectomy: a multicenter comparative study. Fertil Steril. 2006;85(1): $14-21$.

24. Hindley J, et al. MRI guidance of focused ultrasound therapy of uterine fibroids: early results. AJR Am J Roentgenol. 2004;183(6):1713-9.

25. Rabinovici J, et al. Clinical improvement and shrinkage of uterine fibroids after thermal ablation by magnetic resonance-guided focused ultrasound surgery. Ultrasound Obstet Gynecol. 2007;30(5):771-7.

26. Stewart EA, et al. Clinical outcomes of focused ultrasound surgery for the treatment of uterine fibroids. Fertil Steril. 2006;85(1): 22-9. 
27. • Stewart EA, et al. Sustained relief of leiomyoma symptoms by using focused ultrasound surgery. Obstet Gynecol. 2007;110(2 Pt 1): 279-87. Multicenter clinical trial shows sustained symptomatic improvement in about 1/3 of patients one year after MR-guided HIFU of uterine leiomyomas, as well as excellent safety profile of the technique.

28. Fennessy FM, et al. Uterine leiomyomas: MR imaging-guided focused ultrasound surgery-results of different treatment protocols. Radiology. 2007;243(3):885-93.

29. Hesley GK, Gorny KR, Woodrum DA. MR-guided focused ultrasound for the treatment of uterine fibroids. Cardiovasc Interv Radiol. 2013;36(1):5-13.

30. Voogt MJ, et al. Volumetric feedback ablation of uterine fibroids using magnetic resonance-guided high intensity focused ultrasound therapy. Eur Radiol. 2012;22(2):411-7.

31. Venkatesan AM, et al. Magnetic resonance imaging-guided volumetric ablation of symptomatic leiomyomata: correlation of imaging with histology. J Vasc Interv Radiol. 2012;23(6):786-94 e4.

32. Trumm CG, et al. Magnetic resonance imaging-guided focused ultrasound treatment of symptomatic uterine fibroids: impact of technology advancement on ablation volumes in 115 patients. Invest Radiol. 2013;48(6):359-65.

33. •• Rabinovici J, et al. Pregnancy outcome after magnetic resonance-guided focused ultrasound surgery (MRgFUS) for conservative treatment of uterine fibroids. Fertil Steril. 2010;93(1):199-209. Report of 13 sites of the MR-guided focused ultrasound study group shows high rate of successful pregnancy after treatment of uterine leiomyomas.

34. Benagiano G, Habiba M, Brosens I. The pathophysiology of uterine adenomyosis: an update. Fertil Steril. 2012;98(3):572-9.

35. Vercellini P, et al. Adenomyosis: epidemiological factors. Best Pract Res Clin Obstet Gynaecol. 2006;20(4):465-77.

36. Levy G, et al. An update on adenomyosis. Diagn Interv Imaging. 2013;94(1):3-25.

37. Maheshwari A, et al. Adenomyosis and subfertility: a systematic review of prevalence, diagnosis, treatment and fertility outcomes. Hum Reprod Update. 2012;18(4):374-92.

38. Bragheto AM, et al. Effectiveness of the levonorgestrel-releasing intrauterine system in the treatment of adenomyosis diagnosed and monitored by magnetic resonance imaging. Contraception. 2007;76(3):195-9.

39. Smeets AJ, et al. Long-term follow-up of uterine artery embolization for symptomatic adenomyosis. Cardiovasc Interv Radiol. 2012;35(4):815-9.

40. Kim MD, et al. Long-term results of uterine artery embolization for symptomatic adenomyosis. AJR Am J Roentgenol. 2007;188(1):176-81.

41. Grimbizis GF, Mikos T, Tarlatzis B. Uterus-sparing operative treatment for adenomyosis. Fertil Steril. 2014;101(2):472-87.

42. Zhang $X$, et al. Effective ablation therapy of adenomyosis with ultrasound-guided high-intensity focused ultrasound. Int $\mathrm{J}$ Gynaecol Obstet. 2014;124(3):207-11.

43. Fukunishi H, et al. Early results of magnetic resonance-guided focused ultrasound surgery of adenomyosis: analysis of 20 cases. J Minim Invasive Gynecol. 2008;15(5):571-9.

44. - Fukunishi H, et al. Two-year outcome of MRgFUS treatment of adenomyosis. Ultrasound Obstet Gynecol. 2011;38(S1):266. Initial data suggests sustainend symptomatic improvement in about $2 / 3$ of patients two-years after treatment of adenomyosis with MR-guided HIFU.

45. Fan TY, et al. Feasibility of MRI-guided high intensity focused ultrasound treatment for adenomyosis. Eur J Radiol. 2012;81(11):3624-30.

46. Polascik TJ, Mouraviev V. Focal therapy for prostate cancer. Curr Opin Urol. 2008;18(3):269-74.
47. Ahmed HU, Emberton M. Active surveillance and radical therapy in prostate cancer: can focal therapy offer the middle way? World J Urol. 2008;26(5):457-67.

48. Ward JF, Pisters LL. Considerations for patient selection for focal therapy. Ther Adv Urol. 2013;5(6):330-7.

49. Miller DC, et al. Long-term outcomes among localized prostate cancer survivors: health-related quality-of-life changes after radical prostatectomy, external radiation, and brachytherapy. J Clin Oncol. 2005;23(12):2772-80.

50. Holmberg L, et al. A randomized trial comparing radical prostatectomy with watchful waiting in early prostate cancer. New Engl J Med. 2002;347(11):781-9.

51. Mouraviev V, et al. Understanding the pathological features of focality, grade and tumour volume of early-stage prostate cancer as a foundation for parenchyma-sparing prostate cancer therapies: active surveillance and focal targeted therapy. BJU Int. 2011;108(7):1074-85.

52. Meiers I, Waters DJ, Bostwick DG. Preoperative prediction of multifocal prostate cancer and application of focal therapy: review 2007. Urology. 2007;70(6 Suppl):3-8.

53. Karavitakis M, et al. Histological characteristics of the index lesion in whole-mount radical prostatectomy specimens: implications for focal therapy. Prostate Cancer Prostat Dis. 2011;14(1):46-52.

54. Stamey TA, et al. Secondary cancers in the prostate do not determine PSA biochemical failure in untreated men undergoing radical retropubic prostatectomy. Eur Urol. 2001;39(Suppl 4):22-3.

55. Muezzinoglu B, et al. Clinicopathologic significance of multifocal prostate cancer. Lab Invest. 2006;86:151A.

56. Noguchi M, et al. Prognostic factors for multifocal prostate cancer in radical prostatectomy specimens: lack of significance of secondary cancers. J Urol. 2003;170(2 Pt 1):459-63.

57. Coakley FV, et al. Prostate cancer tumor volume: measurement with endorectal MR and MR spectroscopic imaging. Radiology. 2002;223(1):91-7.

58. Chang ST, et al. Endorectal MRI and MR spectroscopic imaging of prostate cancer: developing selection criteria for MR-guided focal therapy. J Magn Reson imaging. 2014;39(3):519-25.

59. Morrow M, Bucci C, Rademaker A. Medical contraindications are not a major factor in the underutilization of breast conserving therapy. J Am Coll Surg. 1998;186(3):269-74.

60. Vaidya JS, et al. Multicentricity of breast cancer: whole-organ analysis and clinical implications. Br J Cancer. 1996;74(5):820-4.

61. Qualheim RE, Gall EA. Breast carcinoma with multiple sites of origin. Cancer. 1957;10(3):460-8.

62. Ahmed HU. The index lesion and the origin of prostate cancer. New Engl J Med. 2009;361(17):1704-6.

63. Arrayeh E, et al. Does local recurrence of prostate cancer after radiation therapy occur at the site of primary tumor? Results of a longitudinal MRI and MRSI study. Int J Radiat Oncol Biol Phys. 2012;82(5):e787-93.

64. van de Ven WJ, Barentsz JO. Prostate cancer: MRI/US-guided biopsy-a viable alternative to TRUS-guidance. Nat Rev Urol. 2013;10(10):559-60.

65. Mouraviev V, Mayes JM, Polascik TJ. Pathologic basis of focal therapy for early-stage prostate cancer. Nat Rev Urol. 2009;6(4):205-15

66. Jayram G, Eggener SE. Patient selection for focal therapy of localized prostate cancer. Curr Opin Urol. 2009;19(3):268-73.

67. Anwar M, et al. Role of endorectal MR imaging and MR spectroscopic imaging in defining treatable intraprostatic tumor foci in prostate cancer: quantitative analysis of imaging contour compared to whole-mount histopathology. Radiother Oncol. 2014;110(2):303-8.

68. Lee HM, Hong JH, Choi HY. High-intensity focused ultrasound therapy for clinically localized prostate cancer. Prostate Cancer Prostatic Dis. 2006;9(4):439-43. 
69. Blana A, et al. High-intensity focused ultrasound for the treatment of localized prostate cancer: 5-year experience. Urology. 2004;63(2):297-300. Large study shows adequate local control of prostate cancer using ultrasound-guided HIFU, but less than optimal safety profile.

70. Vallancien G, et al. Transrectal focused ultrasound combined with transurethral resection of the prostate for the treatment of localized prostate cancer: feasibility study. J Urol. 2004;171(6 Pt 1):2265-7.

71. Ficarra V, et al. Short-term outcome after high-intensity focused ultrasound in the treatment of patients with high-risk prostate cancer. BJU Int. 2006;98(6):1193-8.

72. Tsakiris $P$, et al. Transrectal high-intensity focused ultrasound devices: a critical appraisal of the available evidence. J Endourol. 2008;22(2):221-9.

73. Zini C, et al. Ultrasound- and MR-guided focused ultrasound surgery for prostate cancer. World J Radiol. 2012;4(6):247-52.
74. Lindner $\mathrm{U}$, et al. Focal magnetic resonance guided focused ultrasound for prostate cancer: initial North American experience. Can Urol Assoc Journal (Journal de l'Association des urologues du Canada). 2012;6(6):E283-6.

75. • Napoli A, et al. Real-time magnetic resonance-guided highintensity focused ultrasound focal therapy for localised prostate cancer: preliminary experience. Eur Urol. 2013;63(2):395-8. Initial data demonstrates feasibility MR-guided HIFU of prostate cancer and absence of residual disease in the ablated area on histological analyses of prostatectomy specimens.

76. Chen L, et al. MR-guided focused ultrasound: enhancement of intratumoral uptake of [(3)H]-docetaxel in vivo. Phys Med Biol. 2010;55(24):7399-410.

77. $\mathrm{Mu} \mathrm{Z}$, et al. MR-guided pulsed high intensity focused ultrasound enhancement of docetaxel combined with radiotherapy for prostate cancer treatment. Phys Med Biol. 2012;57(2):535-45. 